\title{
Mutación homocigota en la línea germinal del gen MUTYH en una paciente chilena con poliposis adenomatosa familiar
}

\author{
KARIN ÁLVAREZ ${ }^{1, a}$, MARJORIE DE LA FUENTE ${ }^{1, b}$, \\ PAULINA ORELLANA ${ }^{1,2, b}$, ANA MARÍA WIELANDT ${ }^{1, b}$, \\ CLAUDIO HEINE ${ }^{1}$, CRISTÓBAL SUAZO ${ }^{1}$, UDO KRONBERG ${ }^{1}$, \\ PILAR CARVALLO ${ }^{2, a}$, FRANCISCO LÓPEZ-KÖSTNER ${ }^{1}$
}

\section{Homozygous germline mutation in MUTYH gene in familial adenomatous polyposis}

Recently, MUTYH mutations have been reported to predispose to the development of polyposis. However, polyposis caused by mutations in MUTYH has been characterized as an autosomal recessive hereditary disease, different from the autosomal dominant pattern observed in polyposis caused by APC mutations. We report a 41-year-old female consulting for anemia. Colonoscopy detected multiple sessile polyps and a cecal carcinoma. The patient was operated and in the surgical piece, the tumor invaded serosa and there was lymph node involvement. Approximately 100 polyps were found. The patient received 5 -fluorouracil, as adjuvant therapy. The patient had a sister (of a total of 12 brothers) with a colorectal carcinoma. The genetic study identified a homozygous mutation of the MUTYH gene, called c.340T $>C$, that produces an amino acid change of tyrosine for histidine called p.Y114H. The sister with colorectal cancer was a heterozygous carrier of this mutation.

(Rev Med Chile 2012; 140: 1457-1463).

Key words: Adenomatous polyposis coli; DNA glycosylases; Genes, APC.

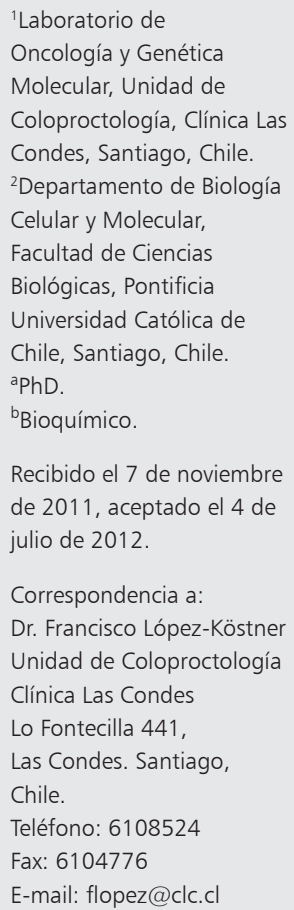

$\mathrm{P}$ oliposis adenomatosa familiar (PAF) es un síndrome hereditario que predispone a un alto riesgo de desarrollo de cáncer colorrectal (CCR). Los pacientes presentan múltiples pólipos (>100) en colon y recto, y de no ser sometidos a una colectomía profiláctica, el 100\% de ellos desarrollará el CCR antes de los 40 años ${ }^{1}$. La base genética de este síndrome fue descubierta por Groden y colaboradores en el año 1991, al determinar que mutaciones en el gen supresor de tumores APC son causantes de esta enfermedad, las cuales presentan una penetrancia cercana al $100 \%$ y un patrón de herencia autosómico dominante ${ }^{2}$. Habitualmente, estos pacientes presentan síntomas desde la segunda década de vida, lo que sumado al antecedente familiar de poliposis y/o CCR, debe despertar la sospecha clínica de PAF. El estudio genético en estos pacientes y la identificación de la mutación en $A P C$ presente en la familia, ofrece una herramienta valiosa para identificar en forma no invasiva aquellos familiares de alto riesgo a desarrollar la enfermedad.

En el año 2002, Al Tassan y colaboradores determinaron que algunos pacientes con poliposis colónica sin mutación identificada en APC, portan mutaciones germinales en ambos alelos del gen $\mathrm{MUTYH}^{3}$. La poliposis asociada a MUTYH, también llamada MUTYH $\underline{\text { Associated }}$ Polyposis (MAP), se caracterizó como una enfermedad hereditaria autosómica recesiva, con un fenotipo indistinguible de $\mathrm{PAF}^{3,4}$. Este gen codifica para una enzima ADN glicosilasa, la cual repara el ADN mutado producto del daño oxidativo causado por especies reactivas de oxígeno ${ }^{5,6}$. La base oxidada 
más estable es la 7,8-dihydroxy-8-oxoguanine (8-oxo-G), la cual aparea con adeninas (A) en lugar de citosinas (C) durante la replicación del $\mathrm{ADN}$, resultando en transversiones genéticas de guanina: citosina por timina: adenina ( $\mathrm{G}$ : $\mathrm{C}>\mathrm{T}$ : A). Normalmente, MUTYH detecta este error y lo repara escindiendo las adeninas mal apareadas. Además, se ha descrito la participación de MUTYH bajo estrés oxidativo, en la activación de vías de muerte celular programada ${ }^{7}$. De esta manera la falta de función de esta enzima aumentaría la tasa de mutaciones somáticas del tipo transversiones, tal como ha sido demostrado en el gen APC y escaparía de la muerte celular programada, permitiendo el desarrollo tumoral ${ }^{3}$.

En un estudio previo en pacientes chilenos con diagnóstico de PAF, se observó que 87,5\% de ellos portan mutaciones en APC (21/24), quedando tres familias sin causa genética identificada ${ }^{8}$. Uno de estos pacientes presenta un aparente patrón de herencia autosómica recesiva, sugerente de la presencia de mutación en MUTYH. Con el fin de identificar alteraciones genéticas en este gen, se realizó el análisis molecular en el paciente índice afectado por poliposis en esta familia.

\section{Pacientes y Métodos}

\section{Caso clínico}

La paciente índice (PAF19) de sexo femenino consultó a los 41 años (2004) por un síndrome anémico. Como parte de su estudio se realizó una endoscopia digestiva alta que no mostró hallazgos patológicos y una colonoscopia completa que demostró la presencia de múltiples pólipos sésiles (más de 100) y pediculados (más de 15), desde el margen anal hasta el ciego. A nivel cecal se evidenció una lesión proliferativa de aproximadamente $2,5 \mathrm{~cm}$ de diámetro mayor, cuya biopsia confirmó un adenocarcinoma tubular moderadamente diferenciado. El diagnóstico preliminar fue de una PAF asociada a un adenocarcinoma cecal. El estudio de etapificación con TAC (tomografía abdominal computarizada) de abdomen y pelvis no mostró signos de diseminación tumoral, realizándose una colectomía total con íleorrectoanastomosis. El estudio anatomopatológico de la pieza quirúrgica mostró una lesión tumoral de 3 $\mathrm{cm}$ de diámetro con invasión hasta la subserosa y compromiso linfonodal. La mucosa del resto de la pieza resecada presentaba un centenar de pólipos sésiles y pediculados, abollonados y lisos de 0,2 a $2 \mathrm{~cm}$ de diámetro mayor. Por tratarse de una paciente en etapa III se realizó un tratamiento con quimioterapia adyuvante en base a 5-fluorouracilo y leucovorina. Hasta la fecha la paciente ha completado 70 meses de seguimiento sin evidencia de enfermedad.

Debido a su historia clínica (poliposis colónica y CCR a edad temprana), la paciente fue derivada al registro de tumores hereditarios para definir el estudio genético apropiado. Este registro incorpora a pacientes con sospecha de enfermedades hereditarias (poliposis adenomatosa familiar, síndrome de Lynch, síndrome de Peutz-Jeghers, entre otros) con la finalidad de educar a los pacientes y familiares respecto a su enfermedad, predisposición de desarrollarla y transmitirla a su descendencia, $\mathrm{y}$ de este modo prevenir el desarrollo del CCR en aquellos familiares asintomáticos.

El análisis de la genealogía de la paciente índice demostró consanguinidad de los padres, sin historia de poliposis o CCR en ellos. Además permitió identificar a una hermana (de un total de 12 hermanos) que presentó CCR (Figura 1), a la que identificamos como PAF585. Ella presentó un cáncer de colon derecho con invasión hasta la subserosa y compromiso linfonodal a los 53 años que fue tratado con cirugía y quimioterapia adyuvante. Un año después, la colonoscopia de control mostró dos nuevos tumores sincrónicos, uno en colon sigmoides a $35 \mathrm{~cm}$ del margen anal y otro cáncer en recto inferior (adenocarcinoma tubular invasor). Cabe destacar que el informe médico de la paciente no registra la presencia de poliposis adenomatosa colónica. La paciente evolucionó tórpidamente falleciendo a los 4 meses posterior a la cirugía debido a un cuadro séptico secundario.

Con respecto a los demás hermanos, cinco de ellos fallecieron en la infancia por accidentes y enfermedades no relacionadas al cáncer. En los cinco hermanos vivos, la paciente índice no declara más casos afectados de poliposis o CCR, sin embargo, desconocemos si ellos se han realizado estudios colonoscópicos.

\section{Estudio genético}

Obtención del ADN genómico desde sangre periférica: el ADN genómico fue aislado a partir de $10 \mathrm{ml}$ de sangre venosa periférica, utilizando el protocolo descrito por Lahiri y Nurnberger $(1991)^{9}$. 
Este ADN fue previamente estudiado en el análisis molecular del gen APC, el cual resultó negativo8.

Análisis mutacional del gen MUTYH: Los 16 exones del gen MUTYH fueron amplificados mediante la técnica de PCR (reacción en cadena de la polimerasa) usando los partidores descritos por Kim y colaboradores, en $2006^{10}$. Reacciones de PCR fueron realizadas en un volumen total de $50 \square$ que contiene $100 \mathrm{ng}$ de ADN genómico, 25 pmoles de cada partidor, $0,2 \mathrm{mM}$ de cada uno de los dNTPs, $1,5 \mathrm{mM} \mathrm{MgCl}$, $1 \mathrm{X}$ del amortiguador suministrado por la enzima y $1 \mathrm{U}$ de la enzima Platinum Taq ADN polimerasa (Invitrogen, Sao Paulo, Brasil). La amplificación fue realizada con el siguiente protocolo: una desnaturación inicial de $1 \mathrm{~min}$ a $95^{\circ} \mathrm{C}$, seguido por 30 ciclos de $30 \mathrm{seg}$ a $95^{\circ} \mathrm{C}, 30$ seg a una temperatura de apareamiento de los partidores (rango $50-58^{\circ} \mathrm{C}$ ), $30 \mathrm{seg}$ a $72^{\circ} \mathrm{C}$, y un paso de extensión final de $10 \mathrm{~min}$ a $72^{\circ} \mathrm{C}$. Los productos amplificados fueron posteriormente analizados mediante dos técnicas de alta sensibilidad para la detección de alteraciones en la secuencia del $\mathrm{ADN}$, que incluye la técnica de SSCP (single strand conformation polymorphism) y secuenciación de $\mathrm{ADN}$. Brevemente, los productos de PCR fueron desnaturados a $95^{\circ} \mathrm{C}$ por 5 min, enfriados en hielo por $5 \mathrm{~min}$, y resueltos por electroforesis en geles de poliacrilamida MDE 0,5X
(FMC Bioproducts), aplicando una corriente de 3 $\mathrm{W}$ a $18^{\circ} \mathrm{C}$ por $12 \mathrm{~h}$. Después de la electroforesis, los geles fueron teñidos con nitrato de plata con el fin de visualizar las bandas de ADN. Los productos de PCR que presentaron una migración anormal en relación al control, fueron secuenciados. Las mutaciones fueron analizadas y confirmadas por dos reacciones de PCR independientes y por secuenciación de las dos hebras de ADN. La mutación se nombró de acuerdo a la nomenclatura propuesta por la Human Genome Variation Society (www. hgvs.org/mutnomen/).

\section{Resultados}

El análisis molecular por SSCP de los 16 exones del gen MUTYH en la paciente índice PAF19, reveló un único patrón de migración anormal presente en el fragmento de ADN que comprende los exones 4 y 5 de este gen (Figura 2). La secuenciación de este fragmento demostró una sustitución nucleotídica de una timina por una citosina presente en ambos alelos (homocigota), localizada en el exón 4, específicamente en el nucleótido 340 según la secuencia descrita para el ARN mensajero de $M U T Y H$, denominada c.340T > C. A nivel de la proteína, esta alteración genética provoca un

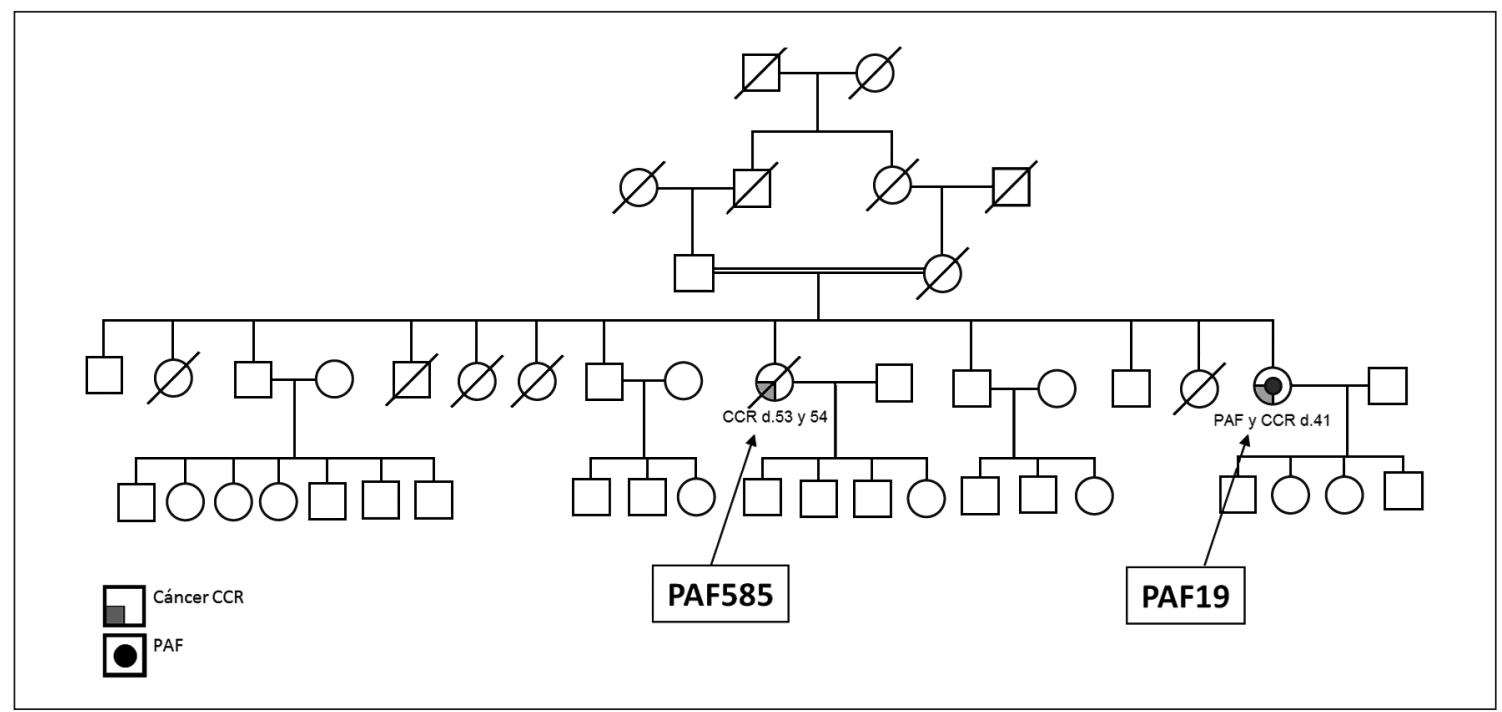

Figura 1. Genealogía de la familia de las pacientes PAF19 y PAF585. Las flechas indican los pacientes a los cuales se les realizó el estudio molecular. Bajo cada sujeto se indica el síndrome, el cáncer y la edad de diagnóstico. O: mujer; $\square$ hombre; $\varnothing \varnothing$ : fallecido; =: consanguinidad. Punto central indica la Poliposis Adenomatosa Familiar (PAF) y el cuadrante inferior izquierdo indica cáncer colorrectal (CCR). 
A.
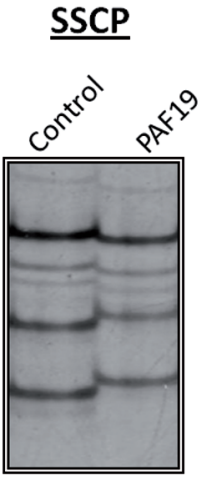

exón $4+5$
B.

Secuenciación

\section{c.340T>C, TAT>CAT, p.Tyr114His}
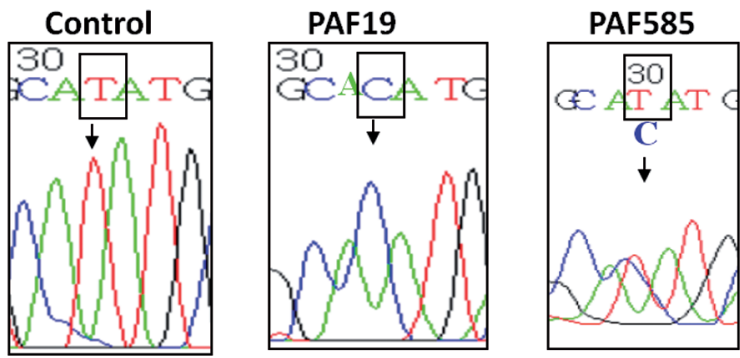

C.

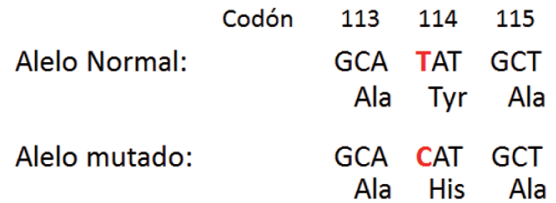

Figura 2. Análisis molecular del gen MUTYH en las pacientes PAF19 y PAF585. En A. se presenta el análisis por SSCP de un fragmento del gen MUTYH que contiene los exónes 4 y 5, realizado a partir del DNA genómico de un individuo control sano y de la paciente PAF19. En la figura se observa el patrón de migración electroforética normal para este fragmento de DNA determinado en la muestra control, en tanto que la paciente PAF19 muestra un patrón de migración alterado. En B. se muestra el resultado de la secuenciación de este fragmento de DNA que contiene los exones 4 y 5, a partir de un individuo control sano, de la paciente PAF19 y paciente PAF585. Las flechas indican la posición nucleotídica alterada. En el individuo control observamos la secuencia de DNA normal que corresponde a una timina, en la paciente PAF19 observamos la sustitución nulceotídica homocigota de la timina por citosina y en la paciente PAF585 observamos la sustitución nucleotídica heterocigota, ya que observamos ambos nucleótidos la timina y citosina en esta posición. En C. se presentan las secuencias normal y mutada de la región que contiene la sustitución nucleotídica. Se muestra la lectura de las secuencias correspondientes a los dos alelos identificados en este estudio, indicando la sustitución nucleotídica de timina por citosina en el codón 114 que conlleva un cambio aminoacídico de Tirosina por Histidina.

cambio aminoacídico de tirosina por histidina en el aminoácido 114, denominada p.Y114H.

Con el fin de determinar la relevancia patogénica del cambio identificado, se evaluó su frecuencia en 100 controles chilenos sanos. Este análisis reveló que esta alteración no se encuentra presente en la población chilena control, lo que sugiere que esta alteración corresponde a una variante alélica de patogenicidad incierta.

Debido a que PAF19 presenta un antecedente familiar de cáncer colorrectal (hermana fallecida PAF585), investigamos si la mutación c.340T > C en MUTYH se encuentra presente en este familiar. Este análisis fue llevado a cabo en el ADN genómico extraído desde el tumor de PAF585 (único material disponible), el cual reveló la presencia de esta alteración en forma heterocigota (Figura 2).

\section{Discusión}

La paciente índice (PAF19) corresponde al único caso de poliposis adenomatosa colónica y CCR en su familia. Su madre falleció a los 81 años y el padre se encuentra vivo con 89 años, ambos sin diagnóstico de la enfermedad. El único antecedente familiar de CCR fue identificado en uno de los once hermanos. Este caso corresponde a una mujer que presentó un cáncer de colon derecho a los 53 años, seguido un año después por dos cánceres presentes en colon sigmoides y recto inferior, falleciendo cuatro meses después por progresión de su enfermedad. De esta manera la familia presenta una sola generación con dos personas afectadas por CCR, lo que concuerda con un patrón de herencia autosómico recesivo (Figura 
1). Cabe destacar que sólo la paciente índice fue diagnosticada con más de 100 pólipos.

El estudio genético molecular de la paciente índice PAF19 se inició con APC, el cual no reveló mutación. Debido a esto se continuó con el estudio molecular del gen MUTYH, tal como lo sugiere la literatura ${ }^{3}$. Este estudio mostró una alteración en MUTYH denominada c.340T > C (p.Y114H), presente en ambos alelos (homocigota). La presencia homocigota de la alteración se debería al antecedente de consanguinidad de los padres.

El cambio encontrado c.340T > C, ha sido previamente descrito por Isidro y colaboradores el año 2004, en una familia portuguesa. En esta familia, la paciente índice y una hermana son portadoras de este cambio, en estado homocigoto. El caso índice fue diagnosticado con menos de 100 pólipos adenomatosos, CCR y cáncer de mama, y su hermana afectada con CCR fue el único antecedente familiar. Como vemos, tanto en nuestra paciente como en el caso descrito en Portugal, las pacientes portadoras del cambio no tienen una historia de transmisión vertical de poliposis y/o cáncer colorrectal, lo que se correlaciona con un modo de herencia autosómico recesivo ${ }^{4,11}$.

Cabe destacar que este cambio no fue identificado en individuos controles sanos de población chilena (100 controles), así como tampoco en población control portuguesa, lo que sugiere un posible efecto patogénico de la alteración genética identificada.

La variante nucleotídica c.340T > C genera un cambio aminoacídico de tirosina por histidina en el codón 114 (p.Y114H), que corresponde a un cambio de un aminoácido polar no cargado a uno positivamente cargado, respectivamente, cuyo efecto funcional no ha sido demostrado. Isidro y colaboradores (2004) realizaron una primera aproximación mediante el alineamiento de la secuencia aminoacídica de la proteína MUTYH humana con otras especies. El análisis demostró que la tirosina 114 es un aminoácido $100 \%$ conservado desde bacteria hasta humano, indicando que este aminoácido es esencial para el correcto funcionamiento de esta enzima reparadora. De esta forma el cambio aminoacídico de tirosina por histidina, en esta posición, podría tener un efecto desfavorable en el plegamiento o en la función de la proteína ${ }^{11}$. Es importante destacar, que un cambio aminoacídico no necesariamente conlleva a la anulación de la función de la proteína, como sí lo haría una proteína trunca. Existen diferentes cambios aminoacídicos que provocan una disminución en distintos niveles de la función, por lo cual estudios que demuestren el efecto de la mutación son relevantes para correlacionarlo con el desarrollo de la enfermedad y su riesgo asociado.

El análisis de cosegregación de la alteración genética en el único familiar afectado por CCR, demostró que PAF585 es portadora del cambio c.340T > C en forma heterocigota. Este hallazgo genera la inquietud sobre el verdadero riesgo de desarrollar CCR en los portadores heterocigotos dentro de la familia. Sin embargo, asumiendo que los padres de la paciente índice son portadores heterocigotos obligados de la alteración, destacamos que ellos a la edad de 81 y 89 años no han desarrollado cáncer colorrectal. De esta manera, son necesarios posteriores estudios para dilucidar la patogenicidad del cambio en MUTYH y así entregar un consejo genético adecuado, en especial a los cuatro hijos de la paciente PAF19, los cuales son portadores obligados del cambio en forma heterocigota.

Con el fin de determinar el riesgo de desarrollar CCR asociado a alteraciones en MUTYH, tanto en forma homocigota como heterocigota, se han realizado estudios de asociación en casoscontroles $^{12-21}$. Estos estudios se han enfocado en las dos alteraciones más frecuentes en MUTYH en la población caucásica, p.Y165C y p.G382D. En estos estudios se determinó que los portadores homocigotos, de ambas variantes, presentan un riesgo mayor de 28 veces de desarrollar CCR, en tanto que los portadores heterocigotos presentan un riesgo 1,34 veces mayor en comparación con la población general ${ }^{22}$. En el presente estudio el cambio identificado es otro, y dada su baja frecuencia en casos y ausencia en población control, no nos es posible realizar estudios de asociación que indiquen el riesgo de desarrollar la enfermedad en los portadores heterocigotos de este cambio.

Con respecto al consejo clínico, a los pacientes que presentan una mutación en ambos alelos de MUTYH y de conocido efecto patogénico, se les recomienda el mismo esquema de seguimiento que a los pacientes con $\mathrm{PAF}^{23}$. Sin embargo, para portadores heterocigotos no existe un consenso en relación al seguimiento, ya que se desconoce su real efecto sobre el desarrollo de la enfermedad. Incluso hay estudios que sugieren que estos individuos tienen el riesgo de la población general 
y que la edad de diagnóstico del cáncer sería similar al cáncer esporádico (70 años $)^{24}$. Debido a esto se recomienda fuertemente la realización de colonoscopias de seguimiento en éstos últimos. La frecuencia del seguimiento debe ser determinada por cada grupo de acuerdo a su experiencia, sin embargo, nos parece adecuado realizar colonoscopias anuales o bianuales al menos hasta los 50 años, hasta no contar con mayor evidencia.

En conclusión, tanto desde el punto de vista del consejo genético como clínico, las mutaciones en el gen MUTYH representan un desafío para el manejo de los pacientes. Se requieren posteriores estudios funcionales para determinar el efecto de las alteraciones que involucran cambios aminoacídicos. Además, se requiere ampliar la caracterización genética de los pacientes y controles, que nos permita determinar el riesgo real de desarrollar el síndrome para los pacientes portadores de alteraciones en este gen. Para ello es necesario seguir profundizando en las bases genéticas y en una caracterización clínica de este síndrome hereditario.

\section{Referencias}

1. Strate Ll, Syngal S. Hereditary colorectal cancer syndromes. Cancer Causes Control 2005; 16: 201-13.

2. Groden J, Thliveris A, Samowitz W, Carlson M, Gelbert $\mathrm{L}$, Albertsen $\mathrm{H}$, et al. Identification and characterization of the familial adenomatous polyposis coli gene. Cell 1991; 66: 589-600.

3. Al-Tassan N, Chmiel NH, Maynard J, Fleming N, Livingston $\mathrm{AL}$, Williams $\mathrm{GT}$, et al. Inherited variants of MYH associated with somatic G:C-->T:A mutations in colorectal tumors. Nat Genet 2002; 30: 227-32.

4. Sampson JR, Dolwani S, Jones S, Eccles D, Ellis A, Evans DG, et al. Autosomal recessive colorectal adenomatous polyposis due to inherited mutations of MYH. Lancet 2003; 362: 39-41.

5. Cheadle P, Sampson JR Exposing the MYtH about base excision repair and human inherited disease. Hum Mol Genet 2003; 12: R159-65.

6. Ohtsubo T, Nishioka K, Imaiso Y, Iwai S, Shimokawa H, Oda $\mathrm{H}$, et al. Identification of human MutY homolog (hMYH) as a repair enzyme for 2-hydroxyadenine in DNA and detection of multiple forms of hMYH located in nuclei and mitochondria. Nucleic Acids Res 2000; 28: 1355-64.

7. Oka S, Nakabeppu Y. DNA glycosylase encoded by MU-
TYH functions as a molecular switch for programmed cell death under oxidative stress to suppress tumorigenesis. Cancer Sci 2011; 102: 677-82.

8. De La Fuente MK, Álvarez KP, Letelier AJ, Bellolio F, Acuña ML, León FS, et al. Mutational screening of the APC gene in Chilean families with familial adenomatous polyposis: nine novel truncating mutations. Dis Colon Rectum 2007; 50: 2142-8.

9. Lahiri DK, Nurnberger JI JR. A rapid non-enzymatic method for the preparation of HMW DNA from blood for RFLP studies. Nucleic Acids Res 1991; 19: 5444.

10. Kim H, Kim HJ, Chi SG, Lee SK, Joo GR, Dong SH, et al. Absence of MutY homologue mutation in patients with multiple sporadic adenomatous polyps in Korea. World J Gastroenterol 2006; 12: 951-5.

11. Isidro G, Laranjeira F, Pires A, Leite J, Regateiro F, Castro E Sousa F, et al. Germline MUTYH (MYH) mutations in Portuguese individuals with multiple colorectal adenomas. Hum Mutat 2004; 24: 353-4.

12. Jenkins MA, Croitoru ME, Monga N, Cleary SP, Cotterchio M, Hopper JL, et al. Risk of colorectal cancer in monoallelic and biallelic carriers of MYH mutations: a population-based case-family study. Cancer Epidemiol Biomarkers Prev 2006; 15: 312-4.

13. Croitoru ME, Cleary SP, Di Nicola N, Manno M, Selander T, Aronson M, et al. Association between biallelic and monoallelic germline MYH gene mutations and colorectal cancer risk. J Natl Cancer Inst 2004; 96: 16314.

14. Peterlongo P, Mitra N, Chuai S, Kirchhoff T, Palmer C, Huang $\mathrm{H}$, et al. Colorectal cancer risk in individuals with biallelic or monoallelic mutations of MYH. Int J Cancer 2005; 114: 505-7.

15. Farrington SM, Tenesa A, Barnetson R, Wiltshire A, Prendergast J, Porteous M, et al. Germline susceptibility to colorectal cancer due to base-excision repair gene defects. Am J Hum Genet 2005; 77: 1.

16. Enholm S, Hienonen T, Suomalainen A, Lipton I Tomlinson I, Kärjä Q, et al. Proportion and phenotype of MYH-associated colorectal neoplasia in a populationbased series of Finnish colorectal cancer patients. Am J Pathol 2003; 163: 827-32.

17. Tenesa A, Campbell H, Barnetson R, Porteous M, Dunlop M, Farrington SM. Association of MUTYH and colorectal cancer. Br J Cancer 2006; 95: 239-42.

18. Wang L, Baudhuin LM, Boardman LA, Steenblock KJ, Petersen GM, Halling KC, et al. MYH mutations in patients with attenuated and classic polyposis and with youngonset colorectal cancer without polyps. Gastroenterology 2004; 127: 9-16.

19. Kambara T, Whitehall VL, Spring KJ Barker MA, Arnold 
$\mathrm{S}$, Wynter CV, et al. Role of inherited defects of MYH in the development of sporadic colorectal cancer. Genes Chromosomes Cancer 2004; 40: 1-9.

20. Webb EL, Rudd MF, Houlston RS. Colorectal cancer risk in monoallelic carriers of MYH variants. Am J Hum Genet 2006; 79: 768-71.

21. Zhou XL, Djureinovic T, Werelius B, Lindmark G Sun QF, Lindblom A, et al. Germline mutations in the MYH gene in Swedish familial and sporadic colorectal cancer. Genet Test 2005; 9: 147-51.

22. Theodoratou E, Campbell H, Tenesa A, Houlston R,
Webb E, Lubbe S, et al A large-scale meta-analysis to refine colorectal cancer risk estimates associated with MUTYH variants. Br J Cancer 2010; 103: 1875-84.

23. Vasen HF, Möslein G, Alonso A, Aretz S, Bernstein I, Bertario L, et al. Guidelines for the clinical management of familial adenomatous polyposis (FAP). Gut 2008; 57: 704-13.

24. Jones N, Vogt S, Nielsen M, Christian D, Wark PA, Eccles D, et al. Increased colorectal cancer incidence in obligate carriers of heterozygous mutations in MUTYH. Gastroenterology 2009; 137: 489-94. 